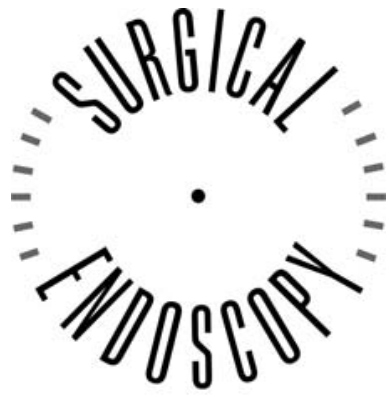

and Other Interventional Techniques

\title{
Laparoscopic parastomal hernia repair is feasible and safe: early results of a prospective clinical study including 55 consecutive patients
}

\author{
B. M. E. Hansson, ${ }^{1}$ I. H. J. T. de Hingh, ${ }^{2}$ R. P. Bleichrodt ${ }^{3}$ \\ ${ }^{1}$ Department of Surgery, Canisius Wilhelmina Hospital, Weg door Jonkerbos 100, Postbus 9015, 6500 GS, Nijmegen, The Netherlands \\ 2 Department of Surgery, Catharina Hospital Eindhoven, Michelangelolaan 2, 5623 EJ, Eindhoven, The Netherlands \\ ${ }^{3}$ Department of Surgery, University Medical Center Nijmegen, Postbus 9101, 6500 HB, Nijmegen, The Netherlands
}

Received: 16 October 2006/Accepted: 2 November 2006/Online publication: 13 March 2007

\begin{abstract}
Background: Parastomal herniation is a common complication, and its operative treatment is notoriously difficult. Recently, the authors have described a laparoscopic technique for closure and reinforcement of the hernia with a hand-made "funnel-shaped" Gore-Tex Dual Mesh. Potentially this technique combines the advantages of a mesh repair with those of minimal invasive surgery.

Methods: In 2002, a multicenter trial of this new technique was started in The Netherlands. To date, 55 consecutive patients ( 27 men; median age, 63 years) with a symptomatic primary $(n=45)$ or recurrent $(n=10)$ parastomal hernia have undergone elective surgery using this technique. The demographic, perioperative, and early follow-up data prospectively collected for these patients are presented in this report.

Results: Of the 55 procedures, $47(85.5 \%)$ could be completed laparoscopically (median operation time, 120 $\mathrm{min}$ ). Conversion to laparotomy was indicated because of dense adhesions prohibiting safe dissection $(n=4)$ or bowel injury $(n=4)$. No in-hospital mortality occurred. Postoperative recovery was uneventful for 47 patients (85\%), who had a median hospital stay of 4 days. Surgical and nonsurgical complications occurred, respectively, for four patients each (7.2\%). Full-thickness enterotomy appeared to be the most troublesome complication. After 6 weeks, when all the patients were reexamined, one recurrence was noted.

Conclusion: Maximal efforts should be undertaken to prevent perioperative full-thickness enterotomy. Because this was achieved for the vast majority of patients,
\end{abstract}

Presented at the 10th World Congress of Endoscopic Surgery, Berlin, Germany on 13-16 September 2006

Correspondence to: B. M. E. Hansson it is concluded that laparoscopic parastomal hernia repair is feasible and safe. Although a longer follow-up period is needed for definitive conclusions to be drawn regarding the recurrence rate, early follow-up evaluation shows very promising results.

Key words: Gore-Tex Dual Mesh — Laparoscopic parastomal hernia repair

Parastomal herniation is a common complication of stoma formation. Its incidence varies significantly, but may be as high as $48 \%$ for colostomies and $28 \%$ for ileostomies [3]. Many operative techniques have been proposed for correction of parastomal hernias, but to date, none has been able to provide satisfactory results, especially in the long term $[3,5]$.

There is growing evidence that herniation in general results from an intrinsic defect in collagen metabolism and wound repair $[9,16]$, and that this together with mechanical factors and a high wound complication rate probably explains the high recurrence rates obtained with techniques relying on primary hernia repair alone $[1,4,5]$. This has resulted in the introduction of prosthetic meshes to correct fascial defects, and randomized clinical studies have indeed proven the superiority of prosthetic meshes in the repair of inguinal [7] and incisional $[2,12]$ hernias, making their usage almost obligatory in these cases.

It seems logical to assume that prosthetic meshes may be of similar value in parastomal hernia repair. However, various authors have published contradictory results on this issue $[6,11,14,15,18]$. This may be explained partly by the differing operative techniques (open or laparoscopic), types of mesh (polypropylene, PTFE, or a combination), and positions of the mesh (intraperitoneal, preperitoneal, or onlay) used [3]. 
Additionally, studies are mostly retrospective in design with only a small number of patients. Therefore, it is currently impossible to draw definitive conclusions on important issues such as perioperative morbidity and mortality and long-term recurrence rates after parastomal hernia mesh repair.

One of the issues raised is the relatively high perioperative complication and mortality rates reaching $65 \%$ and $8 \%$, respectively $[11,13,15,18]$. Recently, we have developed and described a laparoscopic technique for repairing parastomal hernias with a prosthetic mesh [8]. To provide insight into the feasibility and safety of this procedure, a prospective clinical study was started in 2002. The perioperative details and early results for the first 55 consecutive patients included in the study are presented in this report.

\section{Patients and methods}

Between 2002 and 2006, all patients electively referred to the Radboud University Medical Center or the Canisius Wilhelmina Hospital, both in Nijmegen, The Netherlands, with a symptomatic parastomal hernia (severe pain, recurrent obstruction, poor fitting of appliance, cosmetic problems) were asked to participate in this prospective study. Adult patients (ages, 18-80 years) who gave written informed consent were included in the study. The exclusion criteria specified pregnancy, cardiopulmonary contraindications for laparoscopy, or life expectancy shorter than 2 years. Demographic data, indications for enterostomy, comorbidity (chronic obstructive pulmonary disease, cardiovascular disease, and diabetes), American Society of Anesthesiology (ASA) classification, body mass index, size of the hernia, operative details, operation time, perioperative and postoperative complications, time to mobilization, food intake, stoma production, hospital stay, and 6-week follow-up data were recorded on a standard form. The study protocol was reviewed and approved by the institutional ethics commission of the participating hospitals.

\section{Operative procedure}

All procedures were performed or supervised by an experienced laparoscopic surgeon (B.H.), as previously described [8]. For the procedure, the patient is placed under general anesthesia with endotracheal and nasogastric intubation. Intravenous prophylactic antibiotics are given. The patient is placed in a supine position. The surgeon and assistant stand contralateral to the stoma site. The stoma is covered with a sterile finger condom intraluminally and draped with an Opsite drape at skin level for inspection of stoma vascularization and mobilization of the stomaloop during surgery. The Hasson cannula technique is used to create the pneumoperitoneum to a pressure of 12 $\mathrm{mmHg}$. A $30^{\circ}$ laparoscope is inserted, and two working ports are placed under direct vision, creating a triangle with the stoma.

After careful adhesiolysis, hernia contents are reduced, bowel and mesentery are identified, and fascial edges are freed. The hernia opening is narrowed with two Mersilene 0 sutures (Ethicon, Somerville, NJ, USA). A $15 \times 19-\mathrm{cm}$ expanded polytetrafluoroethylene patch (Gore-Tex Dual Mesh Biomaterial, WL Gore \& Associates, Flagstaff, AZ, USA) is fashioned with a central keyhole of $2 \mathrm{~cm}$ and two radial incisions of $5 \mathrm{~mm}$. This makes it possible to give it an intraabdominal funnel-like shape. The mesh is then inserted, unrolled, and tacked to the abdominal wall with titanium tacks (ProTack, Autosuture/Tyco, Norwalk, CT, USA) placed at $1-\mathrm{cm}$ intervals around the circumference of the patch and in the central part around the central hole. The cylindrical part of the mesh forms a collar covering the stoma loop and stitched to the bowel wall with two seromuscular U-stitches using Prolene 3.0 (Ethicon), as shown in Fig. 1.

In this study, serosal bowel lesions were repaired laparoscopically, but in the case of an inadvertent full-thickness enterotomy or when safe dissection was deemed impossible because of dense adhesions, the procedure was converted to an open repair using the same mesh.

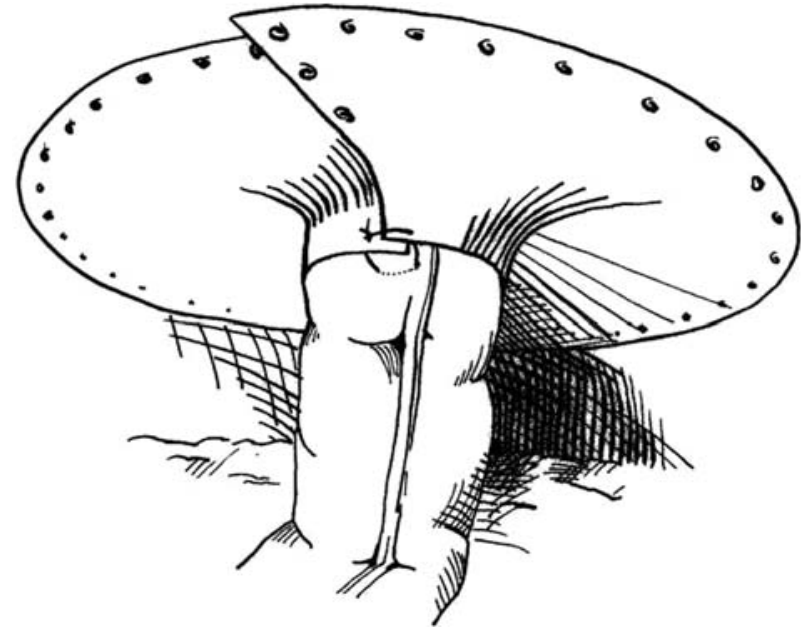

Fig. 1. Operative result for the funnel-shaped Gore-Tex Dual Mesh tacked to the ventral abdominal wall and fixation of the collar with U-stitches.

\section{Postoperative period}

All the patients were examined on a daily basis by the first author. The wound and stoma sites were inspected for signs of infection, formation of seroma, hernia recurrence, or other complications. Unrestricted mobilization and a normal diet were allowed as soon as possible. $\mathrm{Pa}$ tients were discharged when normal mobilization, diet, and stoma production were achieved. All patients were reexamined in the outpatient clinic 6 weeks after the operative procedure.

\section{Results}

\section{Patients}

A total of 55 consecutive patients with a primary $(n=45)$ or recurrent $(n=10)$ parastomal hernia were included in this prospective study. Demographics, ASA classification, and body mass index (BMI) are shown in Table 1 . The stomas were initially constructed for the treatment of colorectal cancer $(n=31)$, fecal incontinence $(n=10)$, inflammatory bowel disease $(n=8)$, congenital anomaly $(n=2)$, acute diverticulitis $(n=1)$, slow-transit constipation $(n=1)$, and perineal trauma $(n=1)$.

\section{Operative procedure}

In all situations, the hernia could be repaired using the Gore-Tex Dual Mesh. Of the 55 procedures, 47 (85.5\%) were completed laparoscopically. Conversion to laparotomy was performed for eight patients (14.5\%). The reasons for conversion were multiple dense adhesions prohibiting safe dissection $(n=4)$ and full-thickness injury to the bowel with contamination $(n=4)$. In three additional cases, serosal bowel damage was noted and repaired laparoscopically.

The median operation time was $120 \mathrm{~min}$ (range, 40$315 \mathrm{~min}$ ), and the median blood loss was $20 \mathrm{ml}$ (range, $0-500 \mathrm{ml}$ ). No significant difference in operation time or blood loss was noted between primary and recurrent hernias (Table 2). Higher incidences of conversion, 
Table 1. Patient demographics and stoma details

\begin{tabular}{lccr}
\hline & Total & Primary hernia & Recurrent hernia \\
\hline$n(\%)$ & $55(100)$ & $45(81,8)$ & $10(18,2)$ \\
Male/female: $n(\%)$ & $27(49.1) / 28(50.9)$ & $24(53.3) / 21(46.7)$ & $3(30) / 7(70)$ \\
Age (years): $n(\%)$ & $63(27-87)$ & $62(27-78)$ & $68(56-87)$ \\
ASA-1: $n(\%)$ & $8(14,5)$ & $7(15,6)$ & $1(10)$ \\
ASA-2: $n(\%)$ & $44(80)$ & $36(80)$ & $8(80)$ \\
ASA-3: $n(\%)$ & $3(5.5)$ & $28(4.4)$ & $1(10)$ \\
BMI $\left(\mathrm{kg} / \mathrm{m}^{2}\right): n$ (range) & $27(19-57)$ & $38(84.4)$ & $25(19-36)$ \\
Colostomy: $n(\%)$ & $47(85.4)$ & $5(11.1)$ & $9(90)$ \\
Ileostomy: $n(\%)$ & $5(9.1)$ & $2(4.4)$ & 0 \\
Urostomy: $n(\%)$ & $3(3.6)$ & $10(4-20)$ & $1(10)$ \\
Diameter hernia sack $(\mathrm{cm}): n$ (range) & $10(4-20)$ & $5(3-10)$ & $8(4-15)$ \\
Trephine diameter $(\mathrm{cm}): n$ (range) & $5(3-10)$ & $39(84,4)$ & $4,5(4-7)$ \\
Not reponible: $n(\%)$ & $43(76.3)$ & $4(40)$ & \\
\hline
\end{tabular}

ASA, American Society of Anesthesiology; BMI, body mass index

Table 2. Operative details and early postoperative complications

\begin{tabular}{lccr}
\hline & Total & Primary hernia & Recurrent hernia \\
\hline OR-Time (min) & $120(40-315)$ & $120(40-315)$ & $120(60-180)$ \\
Blood loss (ml) & $20(0-500)$ & $20(0-500)$ & $25(20-250)$ \\
Conversion (\%) & $8(14.5)$ & $5(11.1)$ & $3(30)$ \\
Intestinal damage (\%) & $6(10.9)$ & $4(8.8)$ & $2(20)$ \\
Reoperation (\%) & $4(7.3)$ & $2(4.4)$ & $2(20)$ \\
Pulmonary complication (\%) & $2(3.6)$ & $2(4.4)$ & 0 \\
Ileus (\%) & $2(3.6)$ & $1(4.4)$ & 0 \\
Mesh infection (\%) & $2(3.6)$ & 0 & $1(10)$ \\
Mortality & 0 & & 0 \\
\hline
\end{tabular}

OR-time $=$ length of the procedure

OR-time and blood loss are given as median values with range

intestinal damage, and reoperation were diagnosed in the recurrent hernia group, but the number of patients was too small for the results to reach statistical significance.

\section{Early postoperative course}

Typically, patients were able to consume a normal diet on day 1 (range, 1-3 days), had stoma production on day 2 (range, $1-13$ days), and could be released from the hospital on postoperative day 4 (range, 2-20 days). No statistically significant differences were observed in these parameters between laparoscopic repairs and converted procedures.

Postoperative complications occurred for eight patients $(14.4 \%)$, with four patients $(7.2 \%)$ requiring a reoperation. One patient underwent reoperation almost immediately after the first operation for correction of bleeding from the epigastric artery. This patient's further recovery was uneventful.

Signs of peritonitis developed in two patients. In one patient, a previously unrecognized full-thickness colonic lesion with fecal contamination of the abdomen was diagnosed during laparotomy on postoperative day 9 . This resulted in removal of the mesh and primary closure of the hernia. In the other patient, a small bowel injury was noted during relaparotomy. After closure of the lesion and mechanical cleaning of the abdomen, the abdomen was closed with the mesh left in place. Both patients received intravenous antibiotics and could be released from hospital, respectively, 14 and 16 days after the initial procedure without signs of infection.

For one patient, the mesh had to be removed on postoperative day 12 because of local abscess formation. Interestingly, this was one of four patients whom required conversion to the open technique because of fullthickness bowel damage.

Nonsurgical complications developed in another four patients (7.2\%). Respiratory insufficiency developed in one patient immediately after surgery, requiring temporary admission to the intensive care unit. Pneumonia developed in another patient, for which treatment with antibiotics was started. Prolonged ileus ( $>7$ days) was noted in two patients. All these patients were treated successfully with conservative measures. No inhospital mortality occurred.

Early wound complications were relatively common but mild. A hematoma was seen at the trocar site in five patients. At the former hernia site, seroma $(n=15)$ and erythema $(n=3)$ were noted, but no signs of infection.

\section{Follow-up evaluation at 6 weeks}

All the patients (100\% follow-up rate) were examined at the outpatient clinic 6 weeks after the initial operation. The majority of the patients $(n=50)$ had an uneventful recovery and were free of symptoms. Four patients re- 
Table 3. Wound complications ${ }^{\mathrm{a}}$

\begin{tabular}{lcl}
\hline & At discharge & After 6 weeks \\
\hline Trocar-site hematoma & 5 & 1 \\
Trocar-site infection & 0 & 0 \\
Stoma-site seroma & 15 & 3 \\
Stoma-site erythema & 3 & 0 \\
Stoma-site infection & 0 & 0 \\
\hline
\end{tabular}

${ }^{a}$ Number of wound complications at discharge and after 6 weeks

ported pain at the site of the mesh. Ulnaropathy of the right arm was diagnosed for one patient.

At the physical examination, one recurrent parastomal hernia was diagnosed in one of the patients for whom conversion to laparotomy was performed because of a full-thickness bowel injury. The hernia was small and did not cause symptoms. Most wound complications had resolved. Only one residual hematoma was noted, and persisting seroma at the site of the hernia was diagnosed for three patients (Table 3).

\section{Discussion}

Parastomal hernia is a common but mostly asymptomatic complication after stoma formation. Mild symptoms include parastomal discomfort, local pain, and obstruction, but these may progress gradually to more severe and even life-threatening complications such as strangulation and perforation. Besides this, parastomal hernias tend to increase in size over time and may result in large disfiguring hernias causing cosmetic problems and poor fitting of the appliance. Fortunately, conservative measures yield satisfactory results for most patients, but surgical repair of the parastomal hernia clearly is indicated for patients with severe complaints.

Many techniques for the repair of parastomal hernias have been described in recent decades. Generally, the techniques fall into one of three categories: local tissue repair, stoma relocation, or repair with prosthetic material [3]. Although clinical trials to compare one technique with the others have never been performed, it is now commonsense to regard techniques using local tissue repair as outdated because of the high recurrence rates in most studies $[1,4,5]$.

Stoma relocation may seem to be an attractive alternative, but it has some major drawbacks such as the risk for the development of a parastomal hernia at the new stoma site and an incisional hernia at the old stoma site $[5,17]$. In addition, this technique requires a formal relaparotomy, causing further damage to the abdominal wall, thereby introducing the risk of an incisional hernia at this particular site. Taking into consideration the recent findings that hernias are, at least in part, caused by underlying defects in wound healing and collagen metabolism $[9,16]$, we consider this technique to be too traumatic for the abdominal wall. Instead, an operative procedure for correction of a parastomal hernia should aim to reinforce the abdominal wall and cause as little additional damage to it as possible. To meet these goals, we previously presented a novel technique in which the parastomal hernia is repaired laparoscopically using a prosthetic mesh.

Meshes for parastomal hernia repair were introduced already 30 years ago, but this has not revolutionized the treatment of parastomal hernias as it has, for instance, changed the treatment of inguinal hernias. This is explained by the concern that, in contrast to inguinal hernia repair, the mesh must be situated in close proximity to the bowel, putting it at risk for adhesive, erosive, and eventually infectious complications. Various authors have indeed reported such problems [14, 19]. However, the choice of the type of mesh seems to be of paramount importance in this respect. Polypropylene meshes were popular in the early days of parastomal mesh repair, but they currently are known to cause dense adhesions and even erosion of the bowel wall. Their usage for parastomal repair is therefore discouraged currently [14, 19].

Despite the abundance of meshes currently available, the "ideal mesh" that should combine rapid ingrowth in the abdominal wall, offer high resistance to infections, and completely lack adhesion to the intestine is not yet available. Meshes made of expanded polytetrafluoroethylene (ePTFE) cause only few adhesions, are soft and pliable, and anchor to the abdominal fascia when fixed with sutures or tacks [10]. Therefore, these meshes are currently deemed most suitable for parastomal hernia repair and were used in the current study.

The practice of performing laparoscopic instead of open repair may be advantageous in terms of surgical damage to the abdominal wall, but it could be argued that it may increase the risk of iatrogenic intestinal laceration because parastomal hernia repair is by definition a reoperation, making disturbed anatomy and multiple dense adhesions very common. Indeed, in the current study, accidental full-thickness enterotomy occurred in six patients $(11 \%)$ despite the presence of an experienced laparoscopic surgeon. However, this still compares favorably with the $19 \%$ rate for inadvertent enterotomies during 270 relaparotomies in open surgery, as reported by van der Krabben et al. [20]. The laparoscopic technique in itself should thus not be regarded as a risk factor for iatrogenic bowel injury. A risk factor may be the presence of a recurrent hernia because the percentage of inadvertent intestinal damage and reoperation is higher for these patients than for those with primary hernias. This may be explained by the disturbed anatomy and fibrosis caused by the previous operations, but it must be realized that the number of patients studied is too small for definitive conclusions to be drawn.

Four of the six bowel perforations were recognized during the initial procedure, which resulted in conversion to an open procedure. In two of these patients, further complications developed in the early postoperative period: an abscess on the mesh requiring its removal on the postoperative day 12 and an early recurrence. In two patients, full-thickness enterotomy was recognized only at the time of relaparotomy for signs of peritonitis, and although the patients eventually recovered completely, the hospital stay was prolonged and one mesh had to be removed because of infection. 
Full-thickness enterotomy should thus be regarded as a very serious perioperative event because it has resulted in considerable postoperative morbidity in affected patients. On the basis of the observations in this study, it might even be argued that mesh repair should be postponed in the event of a recognized enterotomy.

Fortunately, the vast majority of laparoscopic procedures were completed without bowel injury, and the data show that in these cases, the perioperative morbidity rate was very low and postoperative recovery was fast. Wound problems usually were mild and self-limiting. It is not likely that the successful results of the current study are attributable to a favorable patient selection because the patients in this study generally were obese (median BMI, $27 \mathrm{~kg} / \mathrm{m}^{2}$ ), their hernias were large (median hernial sack diameter, $10 \mathrm{~cm}$ ) and mostly not reponible (76\% of cases), and 10 patients with a recurrent hernia were included.

Current knowledge concerning important issues of laparoscopic parastomal hernia repair such as feasibility of the procedure, perioperative morbidity and mortality, the number of mesh infections, and the recurrence rate is sparse and ambiguous. Some authors report low complication and recurrence rates, whereas others are much less optimistic. However, it must be realized that these results are based mainly on case reports and small retrospective series with insufficient follow-up evaluation and quality for definitive conclusions to be drawn on these important issues. The only prospective study addressing the topic of perioperative complication rates reports on only 12 patients, and although the low recurrence rate of only $8 \%$ is promising as compared with other techniques, the perioperative complication rate of $25 \%$ and one fatality may deter others from adopting this technique [11]. Therefore, the current study was undertaken to determine the feasibility and safety of laparoscopic parastomal hernia repair specifically and prospectively.

On the basis of the results from the current study, which represents by far the largest patient series available to date, it is concluded that laparoscopic parastomal hernia repair is feasible, even in cases of recurrent parastomal hernia. However, every possible precaution should be taken to prevent perioperative full-thickness enterotomy because this puts the patient at risk for serious infectious complications in the early postoperative period. In search of answers to other important issues regarding parastomal hernia repair such as infection and recurrence rates in the long term, this group of patients will be closely monitored and results will be reported in the near future.

\section{References}

1. Allen-Mersh TG, Thomson JP (1988) Surgical treatment of colostomy complications. Br J Surg 75: 416-418

2. Burger JW, Luijendijk RW, Hop WC, Halm JA, Verdaasdonk EG, Jeekel J (2004) Long-term follow-up of a randomized controlled trial of suture versus mesh repair of incisional hernia. Ann Surg 240: 578-583

3. Carne PW, Robertson GM, Frizelle FA (2003) Parastomal hernia. Br J Surg 90: 784-793

4. Cassar K, Munro A (2002) Surgical treatment of incisional hernia. Br J Surg 89: 534-545

5. Cheung MT, Chia NH, Chiu WY (2001) Surgical treatment of parastomal hernia complicating sigmoid colostomies. Dis Colon Rectum 44: 266-270

6. de Ruiter P, Bijnen AB (1992) Successful local repair of paracolostomy hernia with a newly developed prosthetic device. Int $\mathrm{J}$ Colorectal Dis 7: $132-134$

7. Grant AM (2002) Open mesh versus nonmesh repair of groin hernia: meta-analysis of randomised trials based on individual patient data. Hernia 6: 130-136

8. Hansson BM, van Nieuwenhoven EJ, Bleichrodt RP (2003) Promising new technique in the repair of parastomal hernia. Surg Endosc 17: 1789-1791

9. Junge K, Klinge U, Rosch R, Mertens PR, Kirch J, Klosterhalfen B, Lynen P, Schumpelick V (2004) Decreased collagen type I/III ratio in patients with recurring hernia after implantation of alloplastic prostheses. Langenbecks Arch Surg 389: 17-22

10. Koehler RH, Begos D, Berger D, Carey S, LeBlanc K, Park A, Ramshaw B, Smoot R, Voeller G (2003) Minimal adhesions to ePTFE mesh after laparoscopic ventral incisional hernia repair: reoperative findings in 65 cases. JSLS 7: $335-340$

11. LeBlanc KA, Bellanger DE, Whitaker JM, Hausmann MG (2005) Laparoscopic parastomal hernia repair. Hernia 9: 140-144

12. Luijendijk RW, Hop WC, van den Tol MP, de Lange DC, Braaksma MM, IJzermans JN, Boelhouwer RU, de Vries BC, Salu MK, Wereldsma JC, Bruijninckx CM, Jeekel J (2000) A comparison of suture repair with mesh repair for incisional hernia. $\mathrm{N}$ Engl J Med 343: 392-398

13. Mancini GJ, Ramshaw BJ, Heniford BT, Novitsky YM, LeBlanc KA, Elieson MJ, Park AE, Kavic SM, Voeller GR, Goldenberg BA (2006) Laparoscopic parastomal hernia repair using a non-slit mesh technique. Surg Endosc 20: S273

14. Morris-Stiff G, Hughes LE (1998) The continuing challenge of parastomal hernia: failure of a novel polypropylene mesh repair. Ann R Coll Surg Engl 80: 184-187

15. Rieger N, Moore J, Hewett P, Lee S, Stephens J (2004) Parastomal hernia repair. Colorectal Dis 6: 203-205

16. Rosch R, Junge K, Knops M, Lynen P, Klinge U, Schumpelick V (2003) Analysis of collagen-interacting proteins in patients with incisional hernias. Langenbecks Arch.Surg 387: 427-432

17. Rubin MS, Schoetz DJ Jr., Matthews JB (1994) Parastomal hernia: is stoma relocation superior to fascial repair? Arch Surg 129: 413-418

18. Safadi B (2004) Laparoscopic repair of parastomal hernias: early results. Surg Endosc 18: 676-680

19. Steele SR, Lee P, Martin MJ, Mullenix PS, Sullivan ES (2003) Is parastomal hernia repair with polypropylene mesh safe? Am J Surg 185: 436-440

20. Van Der Krabben AA, Dijkstra FR, Nieuwenhuijzen M, Reijnen MM, Schaapveld M, Van Goor H (2000) Morbidity and mortality of inadvertent enterotomy during adhesiotomy. Br J Surg 87: 467471 